\title{
Synthetic small peptides acting on B7H1 enhance apoptosis in pancreatic cancer cells
}

\author{
FAN WANG ${ }^{1}$, JUN MA ${ }^{1}$, JUNWEI LIU ${ }^{1}$, HONGCHUAN JIN ${ }^{2}$ and DONGSHENG HUANG ${ }^{1}$ \\ ${ }^{1}$ Department of General Surgery, and ${ }^{2}$ Biomedical Research Center, Sir Run Run Shaw Hospital, \\ Zhejiang University School of Medicine, Hangzhou, Zhejiang 310016, P.R. China
}

Received January 19, 2012; Accepted April 11, 2012

DOI: $10.3892 / \mathrm{mmr} .2012 .970$

\begin{abstract}
The interaction of $\mathrm{B} 7 \mathrm{H} 1$ in tumor cells with programmed death-1 (PD-1) in T cells plays an important role in the suppression of immune responses. However, the effects of the expression of $\mathrm{B} 7 \mathrm{H} 1$ and the PD-1/programmed death 1 ligand 1 (PD-L1) complex on tumor cells themselves remain largely unknown. In order to clarify this, we induced apoptosis in a number of human pancreatic cancer cells with different expression levels of $\mathrm{B} 7 \mathrm{H} 1$ and designed small peptides to interfere with the function of $\mathrm{B} 7 \mathrm{H} 1$. In this study, we chose 2 human pancreatic cancer cell lines (BxPC-3 and Panc-1 cells). Cells with a high expression of B7H1 (BxPC-3 cells and Panc-1 cells treated with interferon- $\gamma$ ) presented much lower levels of drug-induced apoptosis after they were incubated with PD-1 immunoglobulin. Furthermore, the percentage of BxPC-3 apoptotic cells transfected with B7H1 siRNA was higher compared to that of cells transfected with control siRNA. Both of these results indicate that the PD-1/PD-L1 complex transfers a reverse signal to $\mathrm{B} 7 \mathrm{H}^{+}$pancreatic cancer cells upon drug-induced apoptosis in vitro. This effect may be a substantial factor for drug resistance during antitumor therapies. However, synthetic small peptides designed according to the amino acid residues of the PD-1/PD-L1 complex interrupt this effect successfully by their binding to cell membranes and acting like a blocking agent. This result may lead to a breakthrough in pancreatic cancer treatment.
\end{abstract}

Correspondence to: Dr Dongsheng Huang, Department of General Surgery, Sir Run Run Shaw Hospital, Zhejiang University School of Medicine, 3 East Qingchun Road, Hangzhou, Zhejiang 310016, P.R. China

E-mail: dshuang@zju.edu.cn

Abbreviations: Ig, immunoglobulin; PD-1, programmed death-1; CTL, cytotoxic T lymphocyte; IFN, interferon; PE, phycoerythrin; STS, staurosporine; PBS, phosphate-buffered saline; P1, peptide 1; P2, peptide 2; FITC, fluorescein isothiocyanate; FCM, flow cytometry; MTS, 3-(4,5-dimethylthiazol-2-yl)-5-(3-carboxymethoxyphenyl)-2(4-sulfophenyl)-2H-tetrazolium, inner salt; SDS-PAGE, sodium dodecyl sulfate-polyacrylamide gel electrophoresis

Key words: B7H1, apoptosis, pancreatic cancer cell, peptides

\section{Introduction}

Pancreatic cancer is one of the malignancies with very poor prognosis and the 5-year survival rate is approximately $3 \%$ (1). It is regarded as the 8th most common cause of death from cancer worldwide (2), and the 4th leading cause of cancerrelated death in the United States (3). The survival rate has not improved, as shown by studies on gemcitabine-based combination therapy (4). A breakthrough in the treatment of this disease may be required to improve the survival conditions.

B7H1 [CD274, programmed death 1 ligand 1 (PD-L1)] is a B7-related protein with an immunoglobulin (Ig)-like molecule first identified in 1999 (5). Ample evidence confirms that B7H1 is widely expressed in various human gastrointestinal cancers, including pancreatic (6), gastric (7), esophageal (8) and colon (5) cancers, and its expression is constitutive or inducible. B7H1 delivers an inhibitory signal to its receptor, programmed death-1 (PD-1), in T cells, causing the suppression of immune responses (9). The binding of B7H1 and PD-1 occurs through the formation of the PD-1/PD-L1 complex, which makes the 2 proteins interact through the conserved front and side of their $\operatorname{Ig}$ variable $(\mathrm{IgV})$ domains containing some conserved residues (10). Blocking of the B7H1 and PD-1 interaction by neutralizing antibodies restores the cytotoxic $\mathrm{T}$ lymphocyte (CTL)-mediated lysis of tumor cells in vitro (11).

Most studies have focused on the mechanisms of the B7H1 suppressive effect on T cells mediated by PD-1/PD-L1. They have found that mechanisms of action are involved, such as the induction of apoptosis (12) and the exhaustion of T cells (13).

However, in a certain study, mixed $\mathrm{B} 7 \mathrm{H}^{+}$and $\mathrm{B} 7 \mathrm{H}^{-}$cells were cultured together with antigen-specific $\mathrm{CD} 8^{+} \mathrm{CTL}$ in vitro. The researchers did not detect the suppression of the cytolytic function of $\mathrm{CD}^{+} \mathrm{CTL}$ after short-term culture, while they found that $\mathrm{B}^{\mathrm{H}} \mathrm{H}^{-}$cells presented preferential lysis (11). This conclusion suggests that $\mathrm{B} 7 \mathrm{H} 1$ transfers a reverse signal to tumor cells themselves, apart from its role as a ligand to the PD-1 receptor, followed by certain mechanisms that induce the death of $\mathrm{B} 7 \mathrm{H} 1^{+}$cells. To demonstrate this hypothesis, we induced apoptosis in a number of human pancreatic cancer cells with different expression levels of $\mathrm{B} 7 \mathrm{H} 1$ and designed small peptides to interfere with the function of B7H1. Our results indicate that $\mathrm{B} 7 \mathrm{H} 1$ expression in pancreatic cancer cells reduces drug-induced apoptosis and that synthetic small peptides interrupt this inhibition. 


\section{Materials and methods}

Cell lines and cultures. The Panc-1 and BxPC-3 human pancreatic cancer cell lines were obtained from the American Type Culture Collection (ATCC, Manassas, VA, USA). Cells were cultured in RPMI-1640 medium (Gibco, Carslbad, CA, USA) supplemented with $10 \%$ fetal bovine serum (Gibco), $100 \mathrm{U} / \mathrm{ml}$ of penicillin and $100 \mu \mathrm{g} / \mathrm{ml}$ of streptomycin at $37^{\circ} \mathrm{C}$ in a humidified incubator containing $5 \% \mathrm{CO}_{2}$. Treatments were performed after the cells adhered.

Peptide synthesis and treatment. Two small peptides with 6 hydrophilic amino acid residues were synthesized by solid phase synthesis (Hangzhou Angtai Biotech. Co., Ltd., Hangzhou, China). Peptide 1 (P1) was designed according to the amino acid sequence of the connecting site of the PD-1/ PD-L1 complex; Peptide 2 (P2) was synthesized using the similar amino acid residues, but with an uncorrelated sequence as the control. Peptides were lysed in phosphate-buffered saline (PBS) and incubated with the cells at the concentration of $50 \mu \mathrm{g} / \mathrm{ml}$. Fluorescein isothiocyanate (FITC; $5 \mu \mathrm{l}$ )-conjugated P1 and P2 (Angtai Biotechnology) at the concentration of $1 \mathrm{mg} / \mathrm{ml}$ were incubated with BxPC-3 cell samples for flow cytometry (FCM) assay.

FCM assay. BxPC-3 and Panc-1 cells treated with or without interferon (IFN)- $\gamma(500 \mathrm{U} / \mathrm{ml})$ for $24 \mathrm{~h}$ in 6 -well plates were harvested and washed twice with cold PBS. Each sample was incubated with $5 \mu 1$ anti-human B7H1 phycoerythrin (PE), mouse IgG1 isotype control PE (eBioscience, San Diego, CA, USA), FITC-conjugated P1 or control P2 for $30 \mathrm{~min}$ at $4^{\circ} \mathrm{C}$ in the dark. After being washed twice with PBS again, the cells were resuspended in $500 \mu \mathrm{l}$ PBS at the concentration of $1 \times 10^{5} / \mathrm{ml}$ and analyzed by FCM (FACScan; BD Biosciences, Franklin Lakes, NJ, USA). In brief, for apoptosis analysis, cells grown in 6-well plates were pre-treated with P1, P2, recombinant human PD-1 Ig or human IgG (Sino Biological, Beijing, China) at the concentration of $50 \mu \mathrm{g} / \mathrm{ml}$. After $24 \mathrm{~h}$, the cells were treated with staurosporine (STS) at $0.5 \mu \mathrm{M}$ for another $24 \mathrm{~h}$ at $37^{\circ} \mathrm{C}$ and were then collected. After being washed with PBS, the cells were incubated with Alexa Fluor 488 annexin V and propidium iodide (PI) (Invitrogen, Renfrew, UK) for $15 \mathrm{~min}$ at room temperature in the dark, and then the samples were measured by FCM.

siRNA interference. Transfection-related products were all purchased from Santa Cruz Biotechnology, Inc., Santa Cruz, CA, USA. BxPC-3 cells were diluted in fresh medium without antibiotics and transferred to 6 -well plates. Cells grown to a confluence of $50-60 \%$ were transfected with $8 \mu \mathrm{l}$ of B7H1 siRNA or control siRNA per well according to the manufacturer's recommendations. After transfection for $24 \mathrm{~h}$, the efficacy of the siRNA interference was determined by western blot analysis.

MTS assay. 3-(4,5-dimethylthiazol-2-yl)-5-(3-carboxymethoxyphenyl)-2-(4-sulfophenyl)-2H-tetrazolium, inner salt (MTS) assays were performed according to the manufacturer's instructions (CellTiter 96 AQueous Non-Radioactive Cell Proliferation assay; Promega, Madison, WI, USA). Briefly,
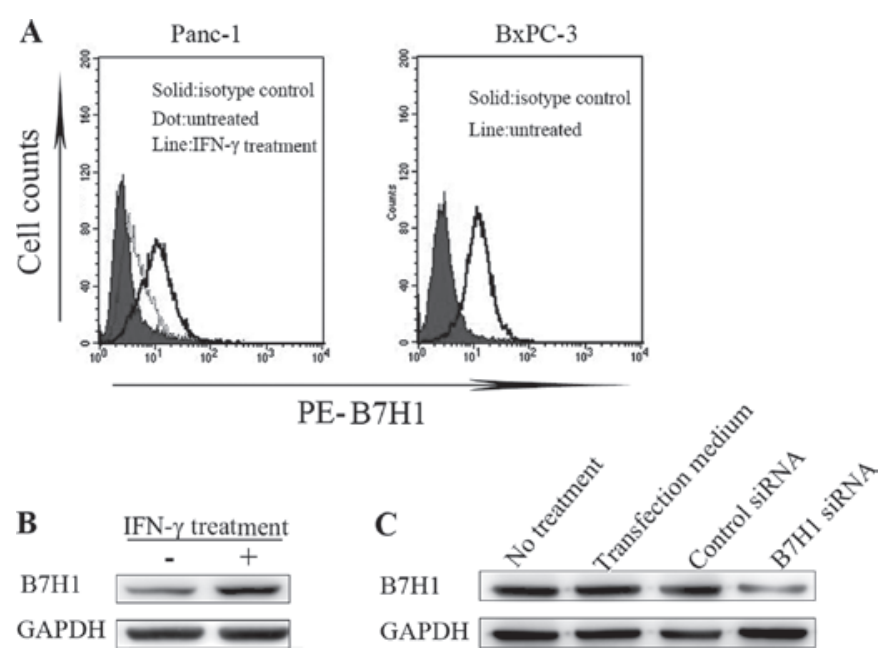

Figure 1. B7H1 was constitutively expressed in Panc-1 cells and highly expressed in BxPC-3 cells. (A) B7H1 expression on the cell membrane was detected by flow cytometry assay using phycoerythrin (PE)-conjugated B7H1 antibody. PE-conjugated mouse immunoglobulin (Ig)G was used as the isotype control. The expression levels of Panc-1 cells treated with or without interferon (IFN)- $\gamma(500 \mathrm{U} / \mathrm{ml})$ for $24 \mathrm{~h}$, and of BxPC-3 untreated cells were compared to the isotype control. (B) Western blot analysis was performed to detect $\mathrm{B} 7 \mathrm{H} 1$ protein levels in the total protein from Panc-1 cells treated or not with IFN- $\gamma$. GAPDH was used as the internal control. (C) B7H1 protein levels in BxPC-3 cells successfully transfected with B7H1 siRNA were compared to 3 controls: normal culture (no treatment), culture with transfection medium and interference with control siRNA. GAPDH was used as the internal control. The data are representative of 3 experiments.

cells were seeded in 96-well culture plates at an optimal density $\left(5 \times 10^{3}\right.$ cells/well) in triplicate wells. After $24 \mathrm{~h}$, the medium was changed and the cells were treated with peptides $(50 \mu \mathrm{g} / \mathrm{ml})$ for another $24 \mathrm{~h}$. Then, STS $(0.5 \mu \mathrm{M})$ was added. After 24 or $48 \mathrm{~h}, 20 \mu \mathrm{l}$ of MTS solution was added to each well (at the total volume of $120 \mu \mathrm{l} / \mathrm{well}$ ) for $2 \mathrm{~h}$ at $37^{\circ} \mathrm{C}$. The absorbance was measured at $570 \mathrm{~nm}$ using a microtitration plate spectrophotometer.

Western blot analysis. To evaluate the expression of the $\mathrm{B} 7 \mathrm{H} 1$ protein and the active degrees of the apoptosis-related protein, poly (ADP-ribose) polymerase 1 (PARP-1), and caspase-3, cellular samples were analyzed by western blot analysis. Cell extracts were prepared with RIPA lysis buffer (Beyotime Biotechnology, Haimen, China). The total protein concentration was measured by the bicinchoninic acid (BCA; Beyotime Biotechnology) method using bovine serum albumin as the standard sample. After the samples were heat-denatured, a total of $40 \mu \mathrm{g}$ of protein samples was subjected to sodium dodecyl sulfate-polyacrylamide gel electrophoresis (SDS-PAGE) (6 or $12 \%$, as required) and transferred to polyvinylidene fluoride membranes (Millipore, Billerica, MA, USA). Membranes were then blocked with 5\% non-fat milk in Tris-buffered solution with $0.5 \%$ Tween-20 (TBST) for $1 \mathrm{~h}$ at room temperature and incubated with primary antibodies specific to B7H1 (R\&D Systems, Minneapolis, MN, USA), PARP-1 and caspase-3 (Epitomics, Burlingame, CA, USA) overnight at $4^{\circ} \mathrm{C}$. After being washed 3 times, membranes were incubated with secondary antibodies (Zhongshan Goldenbridge Biotechnology Co., Beijing, China) or GAPDH (Shanghai Weike Biochemical Reagent Co., Shanghai, China) for $1 \mathrm{~h}$ at room temperature. Signals were detected by 

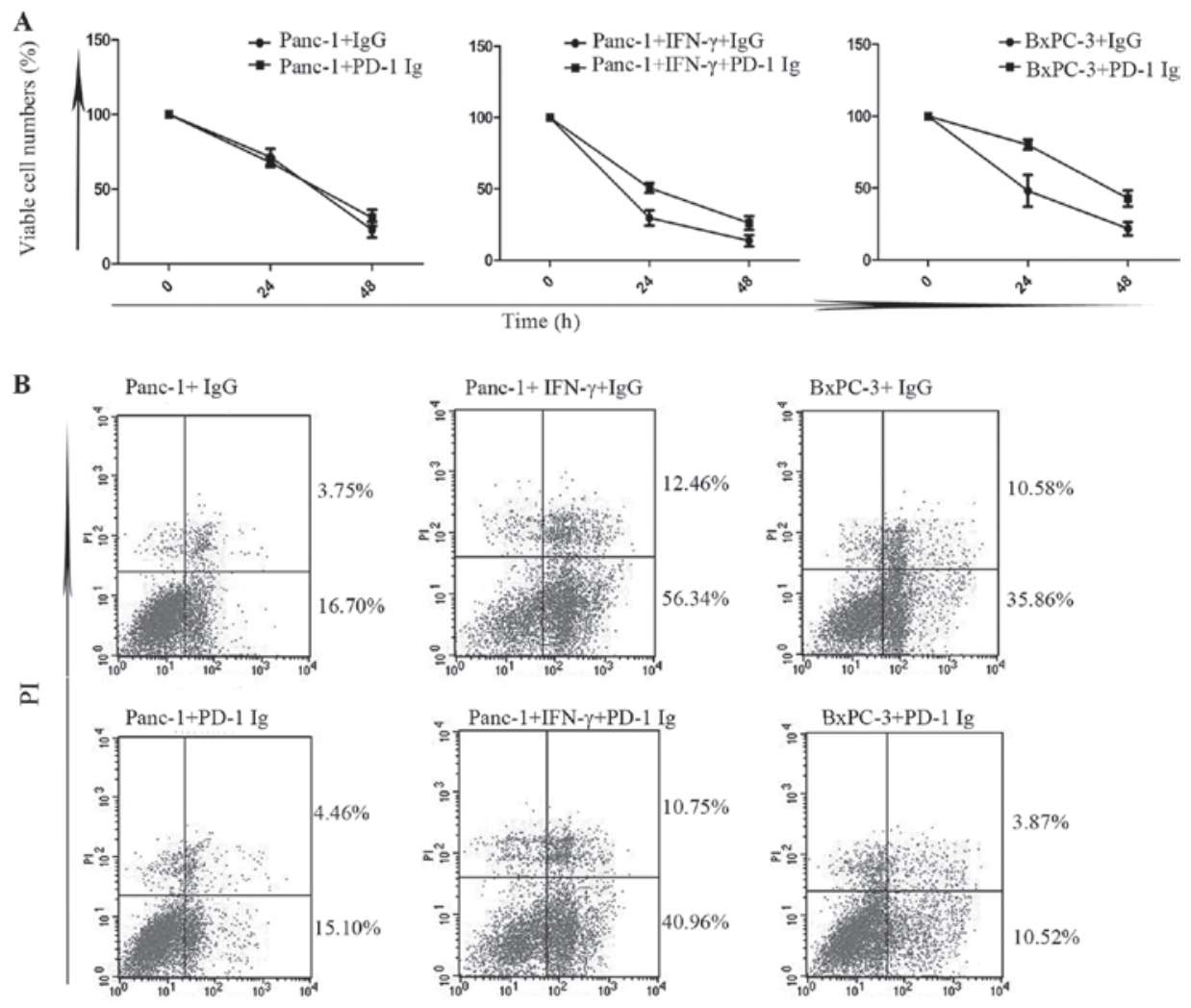

Alexa Fluor 488 annexin V

Figure 2. Binding to programmed death-1 (PD-1) immunoglobulin (Ig) protected tumor cells from staurosporine (STS)-induced apoptosis. Panc-1 cells treated with interferon (IFN)- $\gamma(500 \mathrm{U} / \mathrm{ml}$ ) for $24 \mathrm{~h}$ and BxPC-3 cells incubated with PD-1 Ig or control IgG for $24 \mathrm{~h}$ were treated with STS (0.5 $\mu \mathrm{M})$. (A) MTS assays were used to measure the cell viabilities 24 and $48 \mathrm{~h}$ after STS treatment. Each point is the mean of triplicates with SD. (B) After $24 \mathrm{~h}$ of STS treatment, apoptosis was measured by flow cytometry assays double-stained with Alexa Fluor 488 annexin V and propidium iodide. The data are representative of 3 experiments.

enhanced chemiluminescence detection reagents (Millipore) using ImageQuant LAS-4000 (Fujifilm, Tokyo, Japan). The bands were analyzed using Multi-Gauge software (Fujifilm).

Immunofluorescence. Cells were cultured on microscope slides and washed 3 times with PBS for 15 min before being fixed with $4 \%$ paraformaldehyde at room temperature for $15 \mathrm{~min}$. After being washed $3 \mathrm{z}$ times with PBS for $30 \mathrm{~min}$, the cells were incubated for $2 \mathrm{~h}$ at room temperature with FITC-conjugated P1 or P2. After a further washing step, images were captured on a wide-field fluorescent microscopy (Zeiss, Jena, Germany).

Statistical analysis. All statistical analyses were carried out using the SPSS 19.0 statistical software package. All data were obtained from at least 3 individual experiments. Values are expressed as the means \pm SD. Statistical analysis between groups was performed by one-way ANOVA. A value of $\mathrm{p}<0.05$ was considered to indicate a statistically significant difference.

\section{Results}

B7H1 is constitutively expressed in Panc-1 cells and highly expressed in BxPC-3 cells. We used FCM to determine the levels of $\mathrm{B} 7 \mathrm{H} 1$ protein expression on the cell membrane in BxPC-3 and Panc-1 cells. There was a low-level constitutive expression of B7H1 in Panc-1 cells. This expression was upreg- ulated $24 \mathrm{~h}$ after treatment with IFN- $\gamma(500 \mathrm{U} / \mathrm{ml})$, while B7H1 expression was high in BxPC-3 cells untreated with IFN- $\gamma$ (Fig. 1A). We then extracted the total protein from cells treated as mentioned above to detect the B7H1 expression levels by western blot analysis. The results indicated a similar tendency. Panc-1 cells presented significantly higher levels of $\mathrm{B} 7 \mathrm{H} 1$ expression after treatment with IFN- $\gamma$ (Fig. 1B). The expression levels in BxPC-3 cells were effectively downregulated by siRNA silencing (Fig. 1C). In our study, following B7H1 siRNA transfection, Panc-1 and BxPC-3 cells had a low expression of B7H1, while Panc-1 cells treated with IFN- $\gamma$ and BxPC-3 cells had a high B7H1 expression.

Binding to PD-1 Ig protects tumor cells from STS-induced apoptosis. Panc-1 cells and cells with a high expression of B7H1 were incubated with PD-1 Ig fusion protein $(50 \mu \mathrm{g} / \mathrm{ml})$ or control $\operatorname{IgG}(50 \mu \mathrm{g} / \mathrm{ml})$ for $24 \mathrm{~h}$ after they adhered to 6 -well plates. After extensive washing, the cells were treated with STS $(0.5 \mu \mathrm{M})$ for $24-48 \mathrm{~h}$. MTS assay was used to determine the cell proliferation in the different groups after treatment with STS (Fig. 2A). There was a higher percentage of cell viability in cells with a high expression of $\mathrm{B} 7 \mathrm{H} 1$ pre-treated with $\mathrm{PD}-1$ Ig compared to the controls $(\mathrm{P}<0.05)$, while the differences in Panc-1 cells with low B7H1 expression levels were not significant $(\mathrm{P}>0.05)$. There was an inhibitory action in the cell death caused by STS from the formation of the PD-1/PD-L1 
A

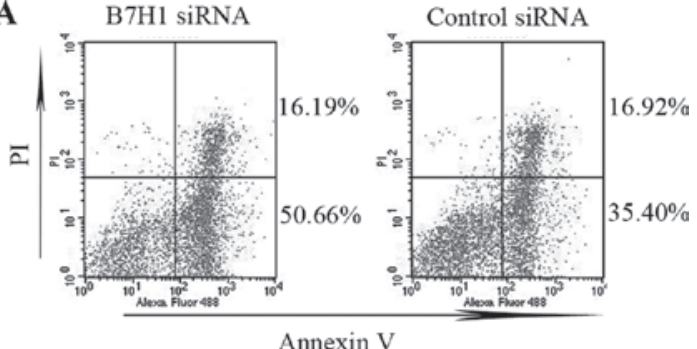

B

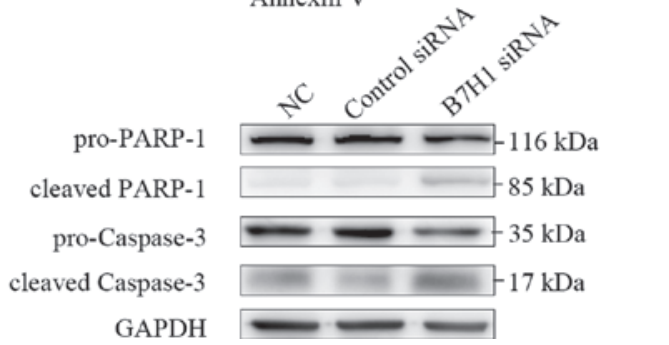

Figure 3. B7H1 knockdown increased apoptosis in BxPC-3 cells. Apoptosis of BxPC-3 cells transfected with B7H1 siRNA or control siRNA was induced by staurosporine (STS) $(0.5 \mu \mathrm{M})$. (A) Apoptosis was measured by flow cytometry assays $24 \mathrm{~h}$ after STS treatment and double-stained with Alexa Fluor 488 annexin $\mathrm{V}$ and propidium iodide. (B) Levels of the apoptosis-related proteins, poly (ADP-ribose) polymerase 1 (PARP-1) and caspase-3, and their cleaved patterns in cells without transfection, transfected with control siRNA and transfected with B7H1 siRNA were analyzed by western blot analysis GAPDH was used as the internal control. The data are representative of 3 experiments.

complex. To explore the mechanisms involved, we then examined cell apoptosis by FCM assay (Fig. 2B). After treatment with STS for $24 \mathrm{~h}$, the percentages of early apoptotic cells in the cells with a high expression of B7H1 pre-treated with PD-1 Ig were significantly lower than those in the controls $(\mathrm{P}<0.05)$ and, as expected, there were no significant differences in the Panc-1 cells $(P>0.05)$. These results suggest that the formation of the PD-1/PD-L1 complex suppresses STS-induced tumor cell apoptosis in cells with a high expression of $\mathrm{B} 7 \mathrm{H} 1$.

B7H1 knockdown increases apoptosis in BxPC-3 cells. In order to examine whether the expression levels of $\mathrm{B} 7 \mathrm{H} 1$ are related to cell apoptosis in BxPC-3 cells, FCM assay and western blot analysis were performed. The percentage of cells at the early stages of apoptosis in cells transfected with B7H1 siRNA was significantly increased compared to the control-transfected cells ( $\mathrm{P}<0.05$; Fig. 3A). Caspase-3 and PARP-1 play key roles during the process of cell apoptosis. We observed marked increases in the cleavage activities of caspase-3 and PARP-1 in the cells transfected with B7H1 siRNA, which indicated the enhancement of apoptosis. This suggests that high levels of B7H1 expression reduce STS-induced tumor cell apoptosis. On the other hand, a successful siRNA interference may increase apoptosis in these cells.

Synthetic peptides designed according to PD-1/PD-L1 complex increase apoptosis in BxPC-3 cells. Synthetic peptide P1 was a small peptide containing 6 amino acid residues. The amino acid sequence, Ser-Asn-Gln-Thr-Asp-Lys, was the same as human PD-1 residues, 73-78, which acted as the connecting site to PD-L1 during the formation of the PD-1/ PD-L1 complex. Peptide P2, with the residues, Ala-Asp-Tyr-
A

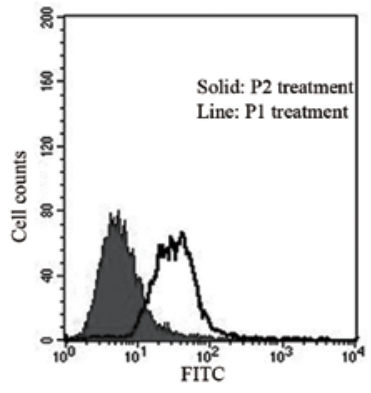

B
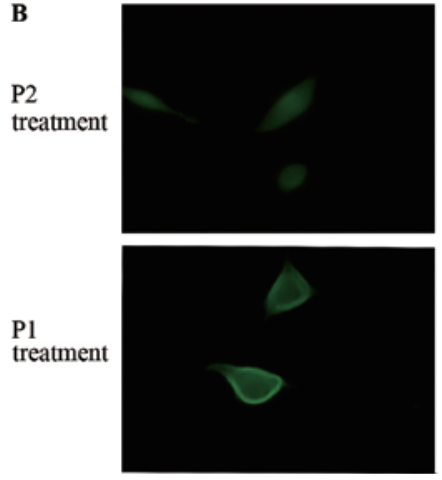

C

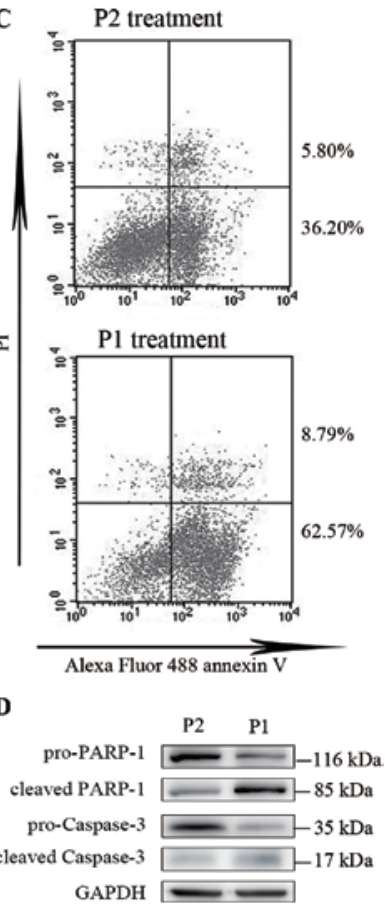

Figure 4. Synthetic peptides designed according to the PD-1/PD-L1 complex increased apoptosis in BxPC-3 cells. (A) Cell samples were incubated with FITC-conjugated peptide 1 (P1) $(5 \mu 1)$ and peptide 2 (P2) $(5 \mu 1)$ for $30 \mathrm{~min}$, then washed with PBS 3 times. Flow cytometry (FCM) assays were used to demonstrate the binding levels. (B) Cells fixed with paraformaldehyde were incubated with FITC-conjugated P1 and P2, and then observed by electron microscopy. (C) Apoptosis of cells pre-treated with P1 or control P2 was induced by staurosporine (STS) $(0.5 \mu \mathrm{M})$ and was measured by FCM assays $24 \mathrm{~h}$ after STS treatment, using the method of double staining with Alexa Fluor 488 annexin $\mathrm{V}$ and propidium iodide. (D) Levels of poly (ADP-ribose) polymerase 1 (PARP-1), caspase- 3 and their cleaved patterns in cells pretreated with P1 and control P2 were analyzed by western blot analysis. GAPDH was used as the internal control. The data are representative of 3 experiments.

Lys-Arg-Ile, was synthesized to act as the control in our study. We used FITC-conjugated P1 and P2 to investigate the efficacy of these synthetic peptides binding to the cell membrane and whether this binding is relative to the expression levels of B7H1. BxPC-3 cell samples incubated with FITC-conjugated synthetic peptides for 30 min were washed extensively and analyzed by FCM. The level of P1 binding was significantly higher than the P2 control (Fig. 4A), which showed a similar tendency in $\mathrm{B} 7 \mathrm{H} 1$ expression. To confirm our result, we performed immunofluorescence analysis to detect whether FITC-P1 binds to the cell membrane effectively. Cells with P1 treatment presented significantly higher fluorescence on the membrane than the control cells (Fig. 4B). With these results, we pre-treated BxPC-3 cells with synthetic peptides and then induced apoptosis by STS, as previously mentioned. In the FCM assays, the cell percentage in the early stages of apoptosis in $\mathrm{P} 1$ pre-treated cells was significantly higher than the P2 control cells ( $\mathrm{P}<0.05$; Fig. $4 \mathrm{C})$. The detection of PARP-1 and caspase-3 showed markedly increasing levels of cleaved proteins in P1 pre-treated cells (Fig. 4D). This may be explained by the interference of the anti-apoptotic effect caused by the PD-1/PD-L1 complex formation, which occurred by the binding of $\mathrm{P} 1$. 


\section{Discussion}

Previous studies have indicated that inhibitory signals are transferred to $\mathrm{T}$ cells by B7H1 in tumor cells, which lead to immune suppression $(11,14,15)$. The blockage of B7H1 and its receptor, $\mathrm{PD}-1$, in $\mathrm{T}$ cells by antibodies improves antitumor immunity (14). Disruption of the B7H1 gene upregulates $\mathrm{T}$ cell responses (16). These studies suggest that $\mathrm{B} 7 \mathrm{H} 1$ and its receptor contribute to tumor cells escaping from immune destruction.

However, in this study, we attempted to explain the mechanism of the resistance to antitumor immunity in $\mathrm{B} 7 \mathrm{H}^{+}$cancer cells from another point of view. The concept that programmed cell death by apoptosis serves as a natural barrier to cancer development has been established by compelling functional studies conducted over the past 2 decades. Yet, another research has revealed how apoptosis is attenuated in tumors that succeed in progressing to states of high-grade malignancy and resistance to therapy (17).

In the present study, we found that B7H1 expressed at a high level inhibits STS-induced cancer cell apoptosis and that the successful B7H1 knockdown increases apoptosis by disrupting this inhibition in the BxPC-3 human pancreatic cell line. A certain study previously demonstrated that $\mathrm{B} 7 \mathrm{H} 1$ siRNA knockdown led to an increase in spontaneous apoptosis, as well as doxorubicin-induced apoptosis in breast cancer cells (18), and another study reported that B7H1 expression in cancer cells plays a role in the induction of the anti-apoptotic mechanism (19). All these results indicate that the expression levels of $\mathrm{B} 7 \mathrm{H} 1$ are related to apoptosis in tumor cells. In due time, this may contribute to the inhibition of $\mathrm{B} 7 \mathrm{H}^{+}$tumor cell lysis in immune responses. However, the underlying molecular mechanisms of the impact are unknown, and further research will help in developing tumor prognosis and therapy.

Furthermore, B7H1 is confirmed to bind its receptor, PD-1, and form the PD-1/PD-L1 complex. It is the structural basis that induces $\mathrm{B} 7 \mathrm{H} 1$ to produce inhibitory effects on immune responses. This complex transfers reverse signals to $\mathrm{B} 7 \mathrm{H} 1^{+}$ tumor cells besides its forward direction to $\mathrm{T}$ cells. This was also detected in our study using PD-1 Ig to mimic the formation of the PD-1/PD-L1 complex. We found that drug-induced apoptosis in cells with a high expression of B7H1 increased significantly after treatment. Since the complex has several conserved domains described previously, small molecule drugs designed to interfere with its inhibitory signal may soon become a reality.

Small peptides less than 8-10 amino acid residues are easily absorbed by the gastrointestinal tract with little degradation. On the other hand, small peptides may cause less side-effects compared to other treatments, such as chemotherapy and radiotherapy. The amino acid residues binding to $\mathrm{B} 7 \mathrm{H} 1$ may interrupt the integrity of the domains and cause some interference. In our study, we designed a small peptide containing 6 amino acid residues to act as a drug. After demonstrating its successful binding to cells with a high expression of $\mathrm{B} 7 \mathrm{H} 1$, we discovered that its binding increased apoptosis in $\mathrm{B} \mathrm{H} 1^{+}$ tumor cells.

In conclusion, our study demonstrates that the high expression of B7H1 and the formation of the PD-1/PD-L1 complex inhibit drug-induced apoptosis in pancreatic cancer cells in vitro. Synthetic small peptides enhance drug-induced apoptosis in pancreatic cancer cells with a high expression of B7H1. We are the first to demonstrate that synthetic small peptides can increase apoptosis in $\mathrm{B} 7 \mathrm{H}^{+}$cancer cells. Our results may lead to a breakthrough in the treatment of pancreatic cancer.

\section{Acknowledgements}

This study was supported by the Key Social Development Project of Major Science and Technology (2011C13036-1). The study was performed at the Biomedical Research Center, Sir Run Run Shaw Hospital, Zhejiang University School of Medicine, Hangzhou, China.

\section{References}

1. Lowenfels AB and Maisonneuve P: Epidemiology and risk factors for pancreatic cancer. Best Pract Res Clin Gastroenterol 20: 197-209, 2006.

2. Jemal A, Siegel R, Ward E, et al: Cancer statistics, 2007. CA Cancer J Clin 57: 43-66, 2007.

3. Jemal A, Siegel R, Ward E, et al: Cancer statistics, 2009. CA Cancer J Clin 59: 225-249, 2009.

4. Merl MY, Li J and Saif MW: The first-line treatment for advanced pancreatic cancer. Highlights from the '2010 ASCO Gastrointestinal Cancers Symposium'. Orlando, FL, USA. January 22-24, 2010. JOP 11: 148-150, 2010.

5. Dong H, Zhu G, Tamada K, et al: B7-H1, a third member of the B7 family, costimulates T-cell proliferation and interleukin-10 secretion. Nat Med 5: 1365-1369, 1999.

6. Nomi T, Sho M, Akahori T, et al: Clinical significance and therapeutic potential of the programmed death-1 ligand/programmed death-1 pathway in human pancreatic cancer. Clin Cancer Res 13: 2151-2157, 2007.

7. Liu SM, Meng Q, Zhang QX, et al: Expression and significance of B7-H1 and its receptor PD-1 in human gastric carcinoma. Zhonghua Zhong Liu Za Zhi 30: 192-195, 2008.

8. Ohigashi Y, Sho M, Yamada Y, et al: Clinical significance of programmed death-1 ligand-1 and programmed death-1 ligand-2 expression in human esophageal cancer. Clin Cancer Res 11: 2947-2953, 2005

9. Chen L: Coinhibitory molecules of the B7-CD28 family in the control of T-cell immunity. Nat Rev Immunol 4: 336-347, 2004.

10. David Y, Yoshimasa T, Masashi I, et al: The PD-1/PD-L1 complex resembles the antigen-binding $\mathrm{Fv}$ domains of antibodies and T cell receptors. PNAS 105: 3011-3016, 2008.

11. Hirano F, Kaneko K, Tamura H, et al: Blockade of B7-H1 and PD-1 by monoclonal antibodies potentiates cancer therapeutic immunity. Cancer Res 65: 1089-1096, 2005.

12. Dong H, Strome SE, Salomao DR, et al: Tumor associated B7-H1 promotes T-cell apoptosis: a potential mechanism of immune evasion. Nat Med 8: 793-800, 2002.

13. Okazaki T and Honjo T: The PD-1-PD-L pathway in immunological tolerance. Trends Immunol 27: 195-201, 2006.

14. Iwai Y, Ishida M, Tanaka Y, et al: Involvement of PD-L1 on tumor cells in the escape from host immune system and tumor immunotherapy by PD-L1 blockade. Proc Natl Acad Sci USA 99: 12293-12297, 2002.

15. Blank C, Brown I, Peterson AC, et al: PD-L1/B7H-1 inhibits the effector phase of tumor rejection by $\mathrm{T}$ cell receptor (TCR) transgenic CD8 ${ }^{+} \mathrm{T}$ cells. Cancer Res 64: 1140-1145, 2004.

16. Latchman YE, Liang SC, Wu Y, et al: PD-L1-deficient mice show that PD-L1 on T cells, antigen presenting cells, and host tissues negatively regulates T cells. Proc Natl Acad Sci USA 101: 10691-10696, 2004.

17. Hanahan D and Weinberg RA: Hallmarks of cancer: the next generation. Cell 144: 646-674, 2011.

18. Ghebeh H, Lehe C, Barhoush E, et al: Doxorubicin downregulates cell surface B7-H1 expression and upregulates its nuclear expression in breast cancer cells: role of B7-H1 as an anti-apoptotic molecule. Breast Cancer Res 12: R48, 2010.

19. Azuma T, Yao S, Zhu G, et al: B7-H1 is a ubiquitous antiapoptotic receptor on cancer cells. Blood 111: 3635-3643, 2008. 